\title{
IMPACTO DE LOS PROGRAMAS DE REHABILITACIÓN CARDÍACA SOBRE LOS FACTORES DE RIESGO CARDIOVASCULAR EN PREVENCIÓN SECUNDARIA
}

\author{
PAULA ANDREA SUÁREZ ${ }^{*}$. ERLING FABIÁN BARRAGÁN ${ }^{2}$. \\ ÓSCAR MAURICIO ÁLVAREZ \\ ${ }^{1}$ Residente de Medicina Física y Rehabilitación. Universidad Militar Nueva Granada. \\ Hospital Militar Central. \\ ${ }^{2}$ Residente de Medicina Física y Rehabilitación. Universidad Militar Nueva Granada, \\ Hospital Militar Central. \\ ${ }^{3}$ Medico Fisiatra. Coordinador programa de Rehabilitación cardíaca Hospital Militar Central. \\ Docente de Medicina Física y Rehabilitación Universidad Militar Nueva Granada. \\ *Correspondencia: paulandreasc@hotmail.com
}

Recibido: Marzo 10 de 2015 Aceptado: Julio 7 de 2015

\begin{abstract}
Resumen
La enfermedad cardiovascular es la primera causa de mortalidad en el mundo en personas mayores de 45 años. En prevención secundaria el adecuado control de los factores de riesgo cardiovascular es el pilar fundamental para disminuir las recurrencias. Los programas de rehabilitación cardíaca han surgido como un mecanismo para controlar dichos factores de riesgo a través del ejercicio y la educación, convirtiéndose en la actualidad en una de las estrategias más costo-efectivas que impactan de manera significativa la morbimortalidad cardiovascular y por todas las causas. Pese a lo anterior, una gran proporción de los pacientes candidatos a realizar estos programas no son referidos, quitandoles la posibilidad de obtener los beneficios sobre su salud. Por esta razón, el proposito de este articulo es realizar una revisión sobre el potencial impacto sobre los factores de riesgo en pacientes con enfermedad cadiovascular conocida y como se pueden beneficiar del acceso formal a programas de rehabilitaciòn cardíaca
\end{abstract}

Palabras Clave: Rehabilitación, enfermedad coronaria, factores de riesgo, prevención, ejercicio aeróbico. 


\title{
IMPACT OF CARDIAC REHABILITATION PROGRAMS ON THE CARDIOVASCULAR RISK FACTORS IN SECONDARY PREVENTION
}

\begin{abstract}
Cardiovascular disease is the leading cause of death among people over 45 years. In secondary prevention the proper control of cardiovascular risk factors is essential to reduce recurrences. Cardiac rehabilitation programs have emerged as a mechanism to control risk factors through exercise and education, becoming at the present one of the most cost-effective strategies that significantly impact cardiovascular morbidity and mortality. However, despite the proven benefits of cardiac rehabilitation programs, most qualified patients are not referred to these and only a minority of eligible patients actually attend these programs. For that reason, the purposes of this article are to review the potential impact of exercise training on cardiovascular risk factors in a person with known cardiovascular disease and as many patients may benefit from formal cardiac rehabilitation programs.
\end{abstract}

Keywords: Rehabilitation, coronary heart disease, risk factors, prevention, aerobic exercise.

\section{O IMPACTO DOS PROGRAMAS DE REABILITAÇÃO CARDÍACA EM FATORES DE RISCO CARDIOVASCULAR EM PREVENÇÃO SECUNDÁRIA}

\begin{abstract}
Resumo
A doença cardiovascular é a primeira causa de mortalidade no mundo em pessoas maiores de 45 anos. O controle adequado dos fatores de risco cardiovascular na prevenção secundaria é o pilar fundamental para reduzir recorrências. Os programas de reabilitação cardíaca têm surgido como um mecanismo para controlar esses fatores de risco através do exercício e a educação, o que agora está se tornando uma das estratégias mais econômicas que impactam significativamente a morbidade e mortalidade cardiovascular por qualquer causa. Não obstante o que antes foi dito, uma grande percentagem dos pacientes eleitos para fazer estes programas não são referidos, tirando deles a possibilidade de obter os benefícios em sua saúde. Portanto, o objetivo do presente artigo é analisar o impacto potencial sobre os fatores de risco em pacientes com doença cardiovascular conhecida $e$ como eles podem se beneficiar de acesso formal aos programas de reabilitação cardíaca.
\end{abstract}

Palavras-chave: Reabilitação, doença cardíaca coronária, fatores de risco, prevenção, exercício aeróbio.

\section{Introducción}

Estudios aleatorizados controlados han mostrado los efectos benéficos del entrenamiento físico en la prevención primaria y secundaria de las enfermedades cardiovasculares (1). De igual manera, múltiples publicaciones muestran resultados estadísticamente significativos sobre los beneficios de los programas de rehabilitación cardíaca basados en entrenamiento físico en pacientes con enfermedad coronaria a través del el control de los factores de riesgo cardiovascular, especialmente después de un evento mayor $(2,3)$. Por lo anterior en la actualidad la Asociación Americana del Corazón considera la rehabilitación cardíaca como uno de los pilares fundamentales para el manejo del paciente cardiovascular con una indicación Clase I y un nivel de evidencia A (4). A pesar de ello, un gran número de pacientes candidatos a ingresar a este tipo de programas no 


\section{Metodología}

Se realizó una búsqueda de la literatura en MEDLINE Y EMBASE para identificar estudios relacionados con programas de rehabilitación cardíaca en prevención secundaria. Se encontraron 27156 referencias relacionadas con los términos MESH de ejercicio terapéutico (exercise therapy), o rehabilitación (rehabilitation) y 203810 con los términos infarto agudo de miocardio (myocardial infarction), enfermedad coronaria (coronary artery disease), síndrome coronario agudo (acute coronary syndrome) intervención coronaria percutánea (percutaneous coronary intervention), angioplastia transluminal (Angioplasty transluminal), Bypass coronario (coronary artery bypass), o cirugía cardíaca (cardiac surgery). La búsqueda se limitó a estudios en humanos, aleatorizados, controlados 0 metanalisis obteniendo 6676 referencias. Los criterios de inclusión fueron: (1) diseño aleatorizado que evaluaran la eficacia de los programas de rehabilitación cardíaca basados en ejercicio; (2) realizados en pacientes con diagnóstico de enfermedad coronaria, (3) bajo la modalidad de ejercicio supervisado o no supervisado en régimen hospitalario y/o ambulatorio; (4) la inclusión de un grupo control no expuesto a ejercicio; (5) que presentaron datos en al menos 1 de los siguientes resultados: mortalidad por cualquier causa, mortalidad cardíaca, morbilidad cardiovascular, revascularización, modificación de factores de riesgo cardiovascular (peso, perfil lipídico, tensión arterial, tabaquismo o factores psicosociales) (6); publicados en Inglés o Español. Se incluyeron en total 65 artículos que cumplían con los criterios de selección. Los hallazgos se describen a continuación.

\section{Programas de rehabilitación cardíaca}

La Organización Mundial de la Salud (OMS) define las enfermedades cardiovasculares como un grupo de desordenes del corazón y los vasos sanguíneos que incluyen la enfermedad coronaria, enfermedad cerebrovascular, enfermedad arterial periférica, enfermedad cardíaca congénita, trombosis venosa profunda y trombo-embolismo pulmonar (6). De acuerdo a las estadísticas publicadas por el Ministerio de la Protección Social en el informe sobre la Situación de salud en Colombia - Indicadores de salud 2007, las enfermedades cardiovasculares fueron la principal causa de muerte tanto en hombres como en mujeres mayores de 45 años representando el $31 \%$ del total de todas las causas (2).

Dentro de las estrategias de prevención secundaria para patologías de origen cardiovascular se encuentran los programas de rehabilitación cardíaca definidos por la OMS como la suma de intervenciones coordinadas $e$ indispensables para que el paciente con enfermedad cardiovascular crónica o post-aguda alcance un funcionamiento social óptimo y logre disminuir o detener la progresión de la enfermedad a través de la promoción de un estilo de vida saludable (7). Se consideran en la actualidad como una de las principales estrategias de intervención que ha demostrado efectividad en múltiples patologías cardiopulmonares, evidenciando una mejoría de la capacidad funcional y la calidad de vida de los pacientes; disminuyendo recurrencias, mejorando síntomas $e$ impactando sobre la morbi-mortalidad de origen cardiovascular y por otras causas (8). Estos resultados se logran a través de estrategias básicas: ejercicio terapéutico, educación y apoyo psicosocial, las cuales cumplen acciones de prevención secundaria y rehabilitación $(9,10)$. En la tabla No.1 se describen los principales beneficios de los programas formales de rehabilitación cardíaca.

Tabla 1. Beneficios de los programas de rehabilitación cardíaca.

\begin{tabular}{lr}
\hline Mejoría del Perfil Lipídico & \\
\hline - Colesterol total & $5 \%$ \\
\hline - Triglicéridos & $15 \%$ \\
\hline - Colesterol HDL & $5-15 \%$ \\
\hline — Colesterol LDL & $2 \%$ \\
\hline Disminución de valores de HbAc1 & $0.8-1 \%$ \\
\hline Disminución valores de tensión arterial & $6 \%$ \\
\hline Mejoría en prevalencia de factores de riesgo psicosocial. (depresión, ansiedad) & \\
\hline Disminución de la Morbilidad y mortalidad cardiovascular & \\
\hline Mejoría en la capacidad Funcional & \\
\hline Reducción en costos hospitalarios &
\end{tabular}

Los protocolos actuales de rehabilitación cardíaca son variados sin embargo, en general tienen una duración de 12 semanas, que consiste en mínimo 3 sesiones de ejercicio semanal, para un total de 36 sesiones. Aunque la mayor parte de la atención va dirigida al componente de ejercicio, la educación de los pacientes y sus familiares sobre su patología y la importancia en la modificación de los factores de riesgo cardiovascular son piedra angular en su desarrollo.

En el año 2004 fallecieron en Colombia 54.270 personas a causa de enfermedades cardiovasculares con una tasa de letalidad del $5 \%$ anual, deduciendo que para dicho año cerca de 1.031 .130 personas se encontraban afectadas por patología de tipo cardiovascular siendo este mismo número candidatas a programas de rehabilitación cardíaca con el fin 
de evitar la progresión de su patología, disminuir la morbimortalidad y los años de vida saludable perdidos (11). Se desconoce el número de pacientes que tuvieron acceso a esta intervención, sin embargo se sabe que el porcentaje de pacientes que ingresan a este tipo de programas es menor al que se encuentra en países desarrollados.

\section{Impacto sobre los valores de perfil lipídico}

El adecuado control de los valores de perfil lipídico ha demostrado ser de importancia en la prevención secundaria de la enfermedad cardiovascular (12). En la actualidad, se recomienda el uso de hipolipemiantes orales aunados a la práctica de ejercicio regular y el seguimiento de una dieta saludable para alcanzar valores adecuados de lipoproteínas de baja densidad (c-LDL) que impidan la progresión de la enfermedad $(4,13)$.

En el estudio DANKUN (14) se estudiaron 104 pacientes con diabetes mellitus tipo 2 o con intolerancia a la glucosa. Un grupo recibió manejo convencional con recomendaciones dietarias; el segundo grupo asistió a un programa de rehabilitación cardíaca en el cual se realizó entrenamiento físico aeróbico, recomendaciones dietarías y generales durante 6 meses. Se observó una reducción significativa en los valores de colesterol LDL (3\%) en los pacientes que asistieron y culminaron el programa de rehabilitación cardíaca, sin observarse variaciones del mismo en el grupo de manejo convencional. Costas Tsakirides y cols., realizaron un estudio en el cual compararon la variación en los factores de riesgo cardiovascular en pacientes diabéticos y no diabéticos sometidos a un programa de entrenamiento físico. Se demostró que los niveles de c-LDL mejoraron significativamente tanto en los pacientes diabéticos como no diabéticos a los 15 meses de seguimiento (15).

Existe evidencia significativa sobre la modificación en otras moléculas del perfil lipídico a través de la práctica de ejercicio. En 2 estudios realizados por Lavie y cols., en pacientes con enfermedad cardiovascular con niveles anormales de lipoproteínas de alta densidad (c-HDL) o triglicéridos y valores adecuados de c- LDL se observó una mejoría del 6\% y 15\% respectivamente al compararlos con los valores iniciales en pacientes sometidos a un programa formal de rehabilitación cardíaca. Durante estos estudios no se utilizaron hipolipemiantes ni otras moléculas que pudieran afectar los resultados $(16,17)$.

\section{Impacto sobre los valores de tensión arterial}

La hipertensión arterial se asocia con un aumento de la mortalidad por todas las causas incluyendo muerte súbita, evento cerebrovascular, falla cardíaca, fibrilación auricular e infarto agudo de miocardio. En la población general la relación entre las complicaciones cardiovasculares y los valores de tensión arterial es lineal en una escala logarítmica. (18) El control efectivo de la tensión arterial posterior a infarto agudo de miocardio reduce la mortalidad cardiovascular en un 20\% (19). Se considera que los pacientes con hipertensión pueden beneficiarse del ejercicio aeróbico de moderada intensidad al disminuir los valores de presión sistólica y diastólica, reducir la resistencia vascular sistémica y modular el sistema neuro-hormonal (20,21).

Sin embargo, los estudios realizados sobre los efectos del ejercicio aeróbico en los valores de presión arterial muestran resultados benéficos aunque variables en el impacto. Se ha observado que durante la práctica de ejercicio aeróbico, la tensión arterial se incrementa durante la fase inicial y es proporcional a la intensidad del esfuerzo (22). El incremento es mayor en los valores de tensión arterial sistólica con apenas una mínima variación en la diastólica o incluso esta última se mantiene en el mismo valor durante la actividad. Una vez terminado el ejercicio se observa una disminución de los valores con respecto a los basales, lo cual puede perdurar por varias horas después de finalizada la actividad. Este efecto hipotensivo del ejercicio ha mostrado ser más significativo en personas con valores tensionales elevados comparados con aquellos normotensos. (23). En este sentido, Fagart y cols. reportaron que el entrenamiento en ejercicio aeróbico disminuyo los valores tanto de tensión sistólica como diastólica en $3 \mathrm{mmHg}$ en personas normotensas, de 6 a 7 $\mathrm{mm} \mathrm{Hg}$ en sujetos con tensión normal alta y entre 8 a 10 $\mathrm{mm} \mathrm{Hg}$ en sujetos con hipertensión arterial (24). En un metaanálisis realizado por este mismo autor, se incluyeron 72 estudios con el fin de evaluar los cambios a nivel de la tensión arterial en pacientes sedentarios normotensos e hipertensos que recibieron un entrenamiento en ejercicio aeróbico. Se observó una reducción de los valores de tensión arterial durante el reposo de 6.9 y $4.9 \mathrm{mmHg}$ en la tensión arterial sistólica y diastólica respectivamente en pacientes hipertensos y 1.9 y $1.4 \mathrm{mmHg}$ para pacientes normotensos al comparar los valores finales con la línea de base (25). Por otra parte, Cornelissen realizó un metaanálisis en el cual evidenció que los pacientes que realizaban un entrenamiento físico aeróbico disminuían la resistencia vascular periférica en un $17 \%$, los niveles de norepinefrina en 29\% y la actividad plasmática de la renina en un $20 \%$ (21).

Teniendo en cuenta los estudios, Fagart y Cornelissen se considera que los mecanismos mediante los cuales se producen estos cambios en las cifras tensionales están relacionados con una disminución en la resistencia vascular periférica, cambios en la respuesta del sistema nervioso 
simpático y la modulación del sistema renina angiotensina aldosterona (26).

\section{Impacto sobre los valores Glucosa}

Las personas con diabetes mellitus tipo 2 tienen al menos el doble de riesgo de muerte prematura, enfermedad coronaria y enfermedad cerebrovascular que aquellos sin esta patología observando una relación lineal entre los niveles de hemoglobina glicosilada y las complicaciones cardiovasculares (27).

Existe una fuerte evidencia del efecto del ejercicio físico sobre la sensibilidad a la insulina y la intolerancia a la glucosa (28), en la prevención de la incidencia de diabetes mellitus tipo 2 en pacientes con intolerancia a la glucosa ya establecida (29) y la reducción de todas las causas de mortalidad y mortalidad cardiovascular en pacientes con diabetes mellitus tipo 2 (30).

Estudios transversales han demostrado que realizar por lo menos 150 minutos semanales de ejercicio aeróbico de moderada intensidad disminuye la prevalencia del síndrome metabólico (31-38). Por otra parte, se ha observado que el ejercicio físico de baja y moderada intensidad tiene efectos favorables sobre los valores de glucosa en sangre en pacientes con diabetes mellitus tipo 2 inmediatamente posterior al ejercicio y dichos efectos se sostienen incluso durante varias horas posterior a la culminación del mismo favoreciendo el nivel de los valores de hemoglobina glicosilada (39). Los efectos son atribuidos a una atenuación de la producción de glucosa hepática y un incremento en la sensibilidad periférica a la insulina así como a la utilización de glucosa a nivel muscular $(40,41)$. Por otra parte, recientemente, se demostró una reducción de la resistencia a la insulina mediante el aumento de los transportadores de glucosa GLUT 4 de la célula muscular, la potenciación de la capacidad oxidativa del musculo esquelético, el aumento del flujo vascular capilar y el incremento de la actividad enzimática $(42,43)$.

Finalmente, los estudios demuestran que los cambios en los estilos de vida incluyendo el ejercicio aeróbico son más efectivos que la metformina para disminuir la incidencia de diabetes mellitus tipo 2 en pacientes con intolerancia a la glucosa. $(44,45)$

\section{Impacto sobre factores psicosociales}

Los factores estresantes psicosociales se han asociado con un aumento del riesgo cardiovascular $(46,47)$. Múltiples estudios indican que los desórdenes emocionales y el estrés crónico son relativamente comunes en pacientes con enfermedad coronaria conocida (48-50). Así mismo, se ha observado una relación estadísticamente significativa entre la depresión y el aumento del riesgo de futuros eventos en pacientes con eventos previos $(51,52)$. Algunos estudios indican que el entrenamiento en ejercicio puede mejorar el perfil psicológico de los pacientes con enfermedad coronaria $(50,53)$. Milani y cols. evaluaron el impacto de los programas de rehabilitación cardíaca sobre la depresión en pacientes con enfermedad cardiovascular con falla cardíaca (fracción de eyección menor del 45\%). Los pacientes con falla cardíaca y depresión inicial que completaron el programa de entrenamiento físico tuvieron un 59\% menos de mortalidad al compararlos con pacientes con depresión y falla cardíaca que no culminaron el entrenamiento $(p=0.04)$ (54). Por otra parte, aunque se ha demostrado que la depresión tiene igual prevalencia en pacientes jóvenes y ancianos con enfermedad cardiovascular, se observa una mayor prevalencia de esta patología en mujeres y en pacientes con diabetes mellitus con diagnóstico de enfermedad coronaria. $(55,56)$. Sin embargo, la respuesta al entrenamiento físico es similar independientemente del sexo, la edad y otras variables demográficas, observándose una disminución mayor al 50\% en la prevalencia de depresión en pacientes que ingresan y culminan los programas formales de rehabilitación cardíaca $(55,56)$.

Otros factores de riesgo psicosocial como la agresividad, ansiedad y aislamiento se han relacionado con el pronóstico cardiovascular $(57,58)$. Lavie y cols realizaron un estudio en pacientes con enfermedad cardiovascular encontrando una prevalencia de ansiedad del 28\% y $14 \%$ en pacientes menores y mayores de 60 años respectivamente. Posterior a un programa de rehabilitación cardíaca basado en ejercicio se observó una disminución en la prevalencia del $61 \%$ y $32 \%$ en cada uno de los grupos (p0.001, p0.01) (55).

Los mecanismos mediante los cuales los factores de riesgo psicosocial aumentan el riesgo cardiovascular aun no son aún muy claros. Sin embargo en la actualidad se consideran que son multifactoriales a través del aumento de otros factores de riesgo cardiovascular los cuales contribuyen directamente a la ateroesclerosis, alteraciones del sistema nervioso autónomo, alteraciones en la reactividad plaquetaria, incremento en la circulación de catecolaminas, vasoreactividad coronaria y vasoconstricción.(59-61). Los programas de rehabilitación cardíaca tienen un efecto directo sobre estos factores y a su vez un efecto indirecto a través de la mejoría en los factores de riesgo psicosocial.

Por otra parte, aunque las intervenciones psicosociales aún no han demostrado una mejoría en el pronóstico de los pacientes con enfermedad coronaria (62), el manejo psicológico continúa siendo una parte integral de los programas de rehabilitación para mejorar la calidad de vida de los pacientes y sus familias (63). 


\section{Tabaquismo}

Pese a los beneficios ampliamente conocidos sobre el cese del tabaquismo, solo la mitad de los pacientes con enfermedades cardiovasculares siguen esta recomendación. En el EUROASPIRE III la prevalencia de tabaquismo persistente en pacientes con enfermedad coronaria fue $51.9 \%$ (64). Por lo anterior, un programa formal de rehabilitación cardíaca debe promover activamente el cese del tabaquismo en los pacientes con enfermedad cardiovascular (65). Los pacientes que continúan fumando después de un infarto agudo de miocardio tienen un riesgo mayor de recurrencia de eventos coronarios (66), pobre control de otros factores de riesgo (67) y pérdida de la calidad de vida (68). Por el contrario, el cese del tabaquismo después de un infarto agudo de miocardio está asociado con una disminución significativa en la mortalidad (69).

En un metaanálisis de estudios de cohorte la combinación OR para muerte en pacientes quienes suspendieron el tabaquismo fue 0.54 (95\% CI: o.42-0:62) y el beneficio fue independiente del sexo, la duración del seguimiento y el sitio de estudio (69).

\section{Morbimortalidad}

Los beneficios de la actividad física sobre la salud han sido demostrados en múltiples estudios. La práctica habitual de ejercicios es uno de los elementos más importantes en la prevención primaria y secundaria de las enfermedades cardiovasculares. Desde esta perspectiva, la rehabilitación cardíaca ha mostrado un impacto significativo en el control de los factores de riesgo cardiovascular (70).

Un metaanálisis de 48 estudios aleatorizados que incluyo 8940 pacientes encontró que la práctica de ejercicio se asocia a una mortalidad más baja por causas cardiovasculares y por todas las causas (odds radio 0.74 y 0.80 respectivamente).Por otra parte, se evidencio un aumento promedio del 33\% del equivalente metabólico y un incremento del 16\% del consumo máximo de oxigeno (71). Otro meta análisis en el cual se incluyeron 63 estudios aleatorizados con 21295 pacientes encontró que la rehabilitación cardíaca reducía las recurrencias de infarto en un $17 \%$ a los 12 meses y redujo la mortalidad hasta un $47 \%$ a los 2 años. Los efectos sobre pronóstico de mortalidad y nuevos eventos fueron mayores para programas que incluían educación en factores de riesgo asociado a un programa de ejercicio estructurado comparado con programas que incluían solo educación en factores de riesgo o consejería sin un componente de ejercicio Los beneficios sobre la mortalidad se incrementaron con un seguimiento más largo con un OR para todas las causas de mortalidad de 0.97 a los 12 meses y 0.53 a los 24 meses (72).

En una revisión sistemática realizada por Taylor y cols. en la cual se analizaron 48 estudios aleatorizados para un total de 8940 pacientes con enfermedad cardiovascular se observó una reducción estadísticamente significativa de la mortalidad por todas las causas y por causas cardiacas $(20 \%$ y $26 \%$ respectivamente) y una reducción del riesgo de infartos no fatales (21\%) (3).

Por otra parte se ha evidenciado una relación dosis respuesta en los resultados de morbilidad y mortalidad cardiovascular. Un estudio realizado por Hammill BG. en el cual se incluyeron más de 30000 pacientes con enfermedad coronaria que participaron en por lo menos 1 sesión de rehabilitación cardíaca. Se observó que aquellos pacientes que completaron 36 sesiones de entrenamiento físico tuvieron un $47 \%$ menos propensión a morir y un $31 \%$ menos de tener infartos agudos de miocardio comparado con aquellos que asistieron a una sola sesión de entrenamiento. (73). A su vez, los beneficios sobre la morbimortalidad son independientes de otras variables. En un estudio realizado por Wannamethee. y cols. en el cual se incluyeron a 772 hombres con enfermedad coronaria en programas de entrenamiento físico se encontró que la actividad física con intensidad leve a moderada se asoció con la disminución de la mortalidad por todas las causas y por causas cardiovasculares independientemente de la edad y otras variables demográficas (74). En el estudio Scottish Surveys los pacientes con enfermedad coronaria que participaron en actividad física de moderada a vigorosa intensidad mostraron una menor mortalidad por todas las causas y mortalidad por causa cardiovascular en un $37 \%$ y $51 \%$ respectivamente independientemente de la edad y el género (75).

Por otra parte, se han documentado que posterior a la realización de procedimientos de revascularización miocárdica percutánea (PCI) los programas de entrenamiento físico han demostrado tener resultados benéficos a largo plazo. Belardinelli y cols. seleccionaron 118 con antecedentes de PCI en los cuales se estudió los episodios de recidivas en los pacientes que realizaron un programa de entrenamiento físico versus aquellos que continuaron con vida sedentaria(76). Los pacientes en el grupo de entrenamiento físico experimentaron menores eventos cardiacos (11.9 Vs 32.2\%) comparados con los pacientes del grupo control en un seguimiento a 33 meses.

Finalmente, Giannuzzi y cols., también demostraron que los pacientes con disfunción ventricular posterior a un infarto agudo de miocardio se benefician del entrenamiento físico al atenuar el proceso de remodelamiento ventricular postinfarto (77). 


\section{Conclusiones}

La revisión de la literatura médica soporta la efectividad de los programas de rehabilitación cardíaca en el control de factores de riesgo cardiovascular. El enfoque de prevención secundaria mejora la calidad de vida de los pacientes y el estatus funcional, reduce los costos de hospitalización, la recurrencia de eventos y la mortalidad a largo plazo. Por lo anterior, promover el ejercicio es uno de los elementos más importantes de los programas de rehabilitación cardíaca. Lo anterior, sumado a los cambios en los estilos de vida que incluyen manejo adecuado de la dieta, toma indicada de medicamentos y manejo de factores psicosociales cumplen un rol fundamental en la reducción de las recurrencias.

El acceso a los programas de rehabilitación cardíaca impacta de manera significativa la morbimortalidad cardiovascular y por todas las causas, siendo una de las estrategias de prevención secundaria más costo-efectivas en la actualidad.

\section{Conflictos de interés}

Los autores declaran no tener de manera directa o indirecta, ningún tipo de conflicto de intereses financieros, académicos o laborales que puedan poner en peligro la validez de este estudio.

\section{Financiaciòn}

La presente revisión fue financiada con recursos propios de los autores.

\section{Referencias}

1. Lavie CJ, Thomas RJ, Squires RW, et al. Exercise training and cardiac rehabilitation in primary and secondary prevention of coronary heart disease. Mayo Clin Proc 2009;84:373-383

2. Departamento Administrativo Nacional de Estadística (DANE) (Colombia). Censo 2005. Causas de defunciones en Colombia 2005. Bogotá. DANE 2005.

3. Taylor RS, Brown A, Ebrahim S, et al. Exercise-based rehabilitation for patients with coronary heart disease: a systematic review and meta-analysis of randomized trials. Am. J. Med. 2004; 116: 682-697.

4. Sidney C. Smith, Jr, Emelia J. Benjamin, Robert O. Bonow, Lynne T. Braun, Mark A. Creager, Barry A. Franklin, et al. AHA/ACCF Secondary Prevention and Risk Reduction Therapy for Patients With Coronary and Other Atherosclerotic Vascular Disease: 2011 Update A Guideline From the American Heart Association and American College of Cardiology Foundation. Circulation 2011;124:2458-2473.

5. Thomas RJ, Houston Miller N, Lamendola C, et al. National survey on gender differences in cardiac rehabilitation programs: patient characteristics and enrollment patterns. $\mathrm{J}$ Cardiopulm Rehabil 1996;16:402-12.

6. Guías Colombianas de Cardiología-Síndrome coronario agudo sin elevación del ST. Revista Colombiana de Cardiología. 2002; Dic 15:3 - 63
7. Dennis L. Kasper, Eugene Braunwald, Anthony S. Fauci, Stephen L.Hauser, Dan L. Longo, J. Larry Jameson, y Kurt J. Isselbacher. Harrison Principios de Medicina Interna: cardiopatia isquemica.16 Ed.Mc Graw Hill.2009

8. Williams MA. Clinical evidence for a health benefit from cardiac rehabilitation: an update. Am Heart J 2006 Nov; 152(5):835-41.

9. Gary J. Balady, MD, FAHA, Chair; Mark A. Williams, PhD, Co-Chair; Philip A. Ades, MD; Vera Bittner, MD, FAHA; Patricia Comoss, RN; JoAnne M. Foody, MD, Core Components of Cardiac Rehabilitation/Secondary Prevention Programs: 2007 Update American Heart Association. J cardiopulm Rehabil Prev. 2007 May-Jun;27(3):121-9.

10. Balady GJ, Williams MA, Ades PA, Bittner V, Comoss P, Foody JM, et al. Southard Core Components of Cardiac Rehabilitation/Secondary Prevention Programs: 2007 Update: A Scientific Statement From the American Heart Association Exercise, Cardiac Rehabilitation, and Prevention Committee, the Council on Clinical Cardiology; the Councils on Cardiovascular Nursing, Epidemiology and Prevention, and Nutrition, Physical Activity, and Metabolism; and the American Association of Cardiovascular and Pulmonary Rehabilitation. Circulation 2007; 115 (20): 2675-82

11. Ministerio de la protección social Universidad de Antioquia facultad nacional de salud pública. Análisis de la situación de salud en colombia, 2002-2007

12. Kannel W. Contributions of the Framingham Study to the conquest of coronary artery disease. Am J Cardiol 1988;62:1109- 12 .

13. Braverman DL: Cardiac rehabilitation: a contemporary review. Am J Phys Med Rehabil 2011;90:599-611.

14. Kannel W. Contributions of the Framingham Study to the conquest of coronary artery disease.Am J Cardiol 1988;62:1109-12.

15. Costas Tsakirides SC. Differential Improvements in Lipid Profiles and Framingham Recurrent Risk Score in Patients With and Without Diabetes Mellitus Undergoing Long-Term Cardiac Rehabilitation. Archives of Physical Medicine and Rehabilitation. 2011 Sep;92:1382-7

16. Lavie CJ, Milani RV. Effects of nonpharmacologic therapy with cardiac rehabilitation and exercise training in patients with low levels of high-density lipoprotein cholesterol. Am J Cardiol 1996;78:1286-89.

17. Cardenas GA, Lavie CJ, Cardenas V, et al. The importance of recognizing and treating low levels of high-density lipoprotein cholesterol: a new era in atherosclerosis management. Rev Cardiovasc Med 2008;9:239-58.

18. Lewington S, Clarke R, Qizilbash N, Peto R, Collins R. Prospective Studies Collaboration. Prospective Studies Collaboration: Age-specific relevance of usual blood pressure to vascular mortality: a meta-analysis of individual data for one million adults in 61 prospective studies. Lancet 2002;360:1903-13.

19. Clark AM, Hartling L, Vandermeer B, et al. Metaanalysis: Secondary prevention programs for patients with coronary artery disease. Ann Intern Med 2005; 143:659-72

20. Hagberg J, Seals D. Exercise training and hypertension. Acta Med Scand 1986;711:131- 6

21. Cornelissen VA, Fagard RH. Effects of endurance training on blood pressure, blood pressure-regulating mechanisms, and cardiovascular risk factors. Hypertension 2005 46: 667-75

22. Fagard R, Amery A. Physical exercise in hypertension. In Laragh J, Brenner B, editors. Hypertension: pathophysiology, diagnosis and management. 2nd ed. New York: Raven Press; 1995. p. 2669-2681.

23. Pescatello LS, Franklin B, Fagard R, et al. American College of Sports Medicine Position Stand: exercise and hypertension. Med Sci Sports Exerc 2004;36:533-53. 
24. Fagard RH. Physical fitness and blood pressure. J Hypertens 1993;11(Suppl 5):S47-52

25. Fagard Rh. Exercise is good for your blood pressure: effects of endurance training and resistance training. Clin Exp Pharmacol Physiol. 2006 Sep;33(9):853-56.

26. Fagard RH. Effect of exercise on blood pressure control in hypertensive patients. Eur J Cardiovasc Prev Rehabil. 2007 Feb;14(1):12-17.

27. Centers for Disease Control and Prevention. National diabetes fact sheet: general information and national estimates on diabetes in the United States, 2000. Atlanta (Ga): U.S. Department of Health and Human Services, Centers for Disease Control and Prevention; 2002.

28. Hu G, Jousilahti P, Barengo NC, et al .Physical activity, cardiovascular risk factors, and mortality among Finnish adults with Diabetes. Diabetes Care 2005; 28: 799-805.

29. Rea TD, Heckbert SR, Kaplan RC, et al. Smoking status and risk for recurrent coronary events after myocardial infarction. Ann Intern Med. 2002 Sep 17;137(6):494-500.

30. Shesneider SH, Amoroso LF. Studies of the mechanism of improve glucose control during exercise in type 2 diabetes mellitus. Diabetologist 1984:26; 355-360.

31. US Department of Health and Human Services. Physical Activity Guidelines Advisory Committee Report 2008. Washington, D.C.; 2008.

32. Halldin M, Rosell M, de Faire U, et al. The metabolic syndrome: prevalence and association to leisure-time and work-related physical activity in 60-year old men and women. Nutr Metab Cardiovasc Dis 2007;17:349-357

33. Lakka TA, Laaksonen DE, Lakka HM, et al. Sedentary lifestyle, poor cardiorespiratory fitness, and the metabolic syndrome. Med Sci Sports Exerc 2003;35:1279-1286.

34. Ford ES, Kohl HW, Mokdad AH, et al. Sedentary behavior, physical activity, and the metabolic syndrome among U.S. adults. Obes Res 2005;13:608-614

35. Carroll S, Cooke CB, Butterly RJ. Metabolic clustering, physical activity and fitness in nonsmoking, middle-aged men. Med Sci Sports Exerc 2000;32:2079-2086.

36. Zhu S, St-Onge M, Heshka S, et al. Lifestyle behaviors associated with lower risk of having the metabolic syndrome. Metabolism 2004;53:1503-1511.

37. Bertrais S, Beyeme-Ondoua JP, Czernichow S et al. Sedentary behaviors, physical activity, and metabolic syndrome in middle-aged French subjects. Obes Res 2005;13:936-944.

38. Irwin ML, Ainsworth BE, Mayer-Davis EJ et al. Physical activity and the metabolic syndrome in a tri-ethnic sample of women. Obes Res 2002;10:1030-1037.

39. Hubinger A, Franzen A, Gries A. Hormonal and metabolic response to physical exercise in hyperinsulinemic and nonhyperinsulinemic type 2 diabetics. Diabetes Res 1987;4:5761

40. Bogardus C, Ravussin E, Robbins D et al. Effects of physical training and diet therapy on carbohydrate metabolism in patients with glucose intolerance and non-insulin dependent diabetes mellitus. Diabetes 1984;33:311-8.

41. Devlin J, Hirshman M, Horton E, et al. Enhanced peripheral and splanchnic insulin sensitivity in NIDDM men after a single bout of exercise. Diabetes 1987;36:434-9.

42. Harding $\mathrm{AH}$, Williams De. Is the association between dietary fat intake and insulin resistance modified by physical activity? Metabolis, 2002;50:1186-1192

43. Sigal RJ, Kenny GP. Exercise and Diabetes Mellitus. Pick up JC. William C. textbook of diabetes. 3ra ed. Oxford Backwell Science 2003

44. Knowler WC, Barrett-Connor E, Fowler SE, et al. Diabetes Prevention Program Research Group. Reduction in the incidence of type 2 diabetes with lifestyle intervention or metformin. NEngl J Med 2002;346:393-403
45. Tuomilehto J, Lindström J, Eriksson JG, et al. Finnish Diabetes Prevention Study Group. Prevention of type 2 diabetes mellitus by changes in lifestyle among subjects with impaired glucose tolerance. NEngl J Med 001;344:1343-50

46. Rozanski A, Blumental JA, Davidson KW, et al. The epidemiology, pathophysiology, and management of psychosocial risk factors in cardiac practice: the emerging field of behavioral cardiology. J Am Coll Cardiol 2005;45:637651.

47. Lavie CJ, Milani RV, Lavie TJ. Impact of cardiac rehabilitation, exercise training, and fitness on psychological distress. In: Sher L, editor. Psychological factors and cardiovascular disorders: the role of psychiatric pathology \& maladaptive personality features. Haup- pauge (NY): Nova Science Publishers; 2009. p. 312-329.

48. Rozanski A, Blumenthal JA, Davidson KW, Saaob PG, Kubzansky L. The epidemiology, pathophysiology, and management of risk factors in cardiac practice. J. Am. Coll. Cardiol. 45, 637-651 (2005).

49. Dimsdale JE. Psychological stress and cardiovascular disease. J Am Coll Cardiol 2008;51:1237-46.

50. Lichtman JH, Bigger T, Blumenthal JA et al. Depression and coronary artery disease. Recommendations for screening, referral, and treatment. A Science Advisory from the American Heart Association Prevention Committee of the Council on Cardiovascular Nursing, Council on Clinical Cardiology, Council on Epidemiology and Prevention, and Interdisciplinary Council on Quality of Care and Outcome Research. Circulation 2008;118: 1768-75.

51. Esperance F, Frasure-Smith N, Talajic M, et al. Five-year risk of cardiac mortality in relation to initial severity and one-year changes in depression symptoms after myocardial infarction. Circulation 2002;105:1049-1053.

52. Rozanski A, Blumenthal JA, Kaplan J. Impact of psychological factors on the pathogenesis of cardiovascular disease and implications for therapy. Circulation 1999;99:2192-2217

53. Milani RV, Lavie CJ. Impact of cardiac rehabilitation on depression and its associated mortality. Am. J. Med. 2007; 120: 799-806.

54. Milani RV, Lavie CJ, Mehra MR, et al. Impact of exercise training and depression on survival in heart failure due to coronary heart disease. Am J Cardiol 2011;107:64-68

55. Lavie CJ, Milani RV. Adverse psychological and coronary risk profiles in young patients with coronary artery disease and benefits of formal cardiac rehabilitation. Arch Intern Med 2006; $166: 1878-83$

56. Lavie CJ, Milani RV. Cardiac rehabilitation and exercise training programs in metabolic syndrome and diabetes. $\mathrm{J}$ Cardiopulm Rehabil 2005;25:59-66

57. Dimsdale JE. Psychological stress and cardiovascular disease. J Am Coll Cardiol 2008;51:1237-1246

58. Das S, O'Keefe JH. Behavioral cardiology: recognizing and addressing the profound impact of psychosocial stress on cardio-vascular health. Curr Atheroscler Rep 2006;8:111118.

59. Lavie CJ, Milani RV. Prevalence of hostility in young coronary artery disease patients and effects of cardiac rehabilitation and exercise training. Mayo Clin Proc 2005;80:335-342

60. Milani RV, Lavie CJ, Cassidy MM. Effects of cardiac rehabilitation and exercise training programs on depression in patients after major coronary events. Am Heart J 1996;132:726-732

61. Milani RV, Lavie CJ. Impact of cardiac rehabilitation on depression and its associated mortality. Am J Med 2007;120:799-806

62. Milani RV, Lavie CJ. Impact of cardiac rehabilitation on depression and its associated mortality. Am. J. Med 2007; 120: 799-806. 
63. Sidney C. Smith, Jr, Emelia J. Benjamin, Robert O. Bonow, Lynne T. Braun, Mark A Guideline From the American Heart Association and American College of With Coronary and Other Atherosclerotic Vascular Disease: 2011 Update : A Guideline From the American Heart Association and American College of Cardiology Foundation. Circulation 2011, 124:2458-2473

64. Kotseva K, Wood D, De Backer G et a. EUROASPIRE Study Group: EUROASPIRE III: a survey on the lifestyle, risk factors and use of cardioprotective drug therapies in coronary patients from 22 European countries. Eur J Cardiovasc Prev Rehabil. 2009 Apr; 16(2):121-37.

65. Kallio V, Hamalainen H, Hakkila J, et al. Reduction in sudden death by a multifactorial intervention program after acute myocardial infarction. Lancet 1979;2:1091-4

66. Rea TD, Heckbert SR, Kaplan RC et al. Smoking status and risk for recurrent coronary events after myocardial infarction. Ann. Intern. Med 2002; 137(6):494-500.

67. Rallidis LS, Panagiotakos DB, Pitsavos C, Stefanadis C, Kremastinos DT. Persistent smokers after myocardial infarction: a group that requires special attention. Int. J. Cardiol.2005; 100(2): 241-245.

68. Taira DA, Seto TB, Ho KK et al. Impact of smoking on healthrelated quality of life after percutaneous coronary revascularization. Circulation 2000; 102: 1369-74

69. Wilson K, Gibson N, Willan A, Cook D. Effect of smoking cessation on mortality. Meta-analysis of cohort studies. Arch. Int. Med 2000;160: 939-44.

70. Roger VL, Go AS, Lloyd-Jones DM, et al. Heart disease and stroke statistics 2011 update: A report from the American Heart Association. Circulation. 2011 Feb 1;123(4):e18-e209.

71. Lawler PR, Filion KB, Eisenberg MJ. Efficacy of exercise-based cardiac rehabilitation post-myocardial infarction: A systematic review and meta-analysis of randomized controlled trials. Am Heart J. 2011 Oct; 162(4):571-584.

72. Clark AM, Hartling L, Vandermeer B, et al. Metaanalysis: Secondary prevention programs for patients with coronary artery disease. Ann Intern Med 2005; 143:659-72

73. Hammill BG, Curtis LH, Schulman KA, et al: Relationship between cardiac rehabilitation and long term risks of death and myocardial infarction among elderly Medicare beneficiaries. Circulation. 2010 Jan 5;121(1):63-70

74. Wannamethee SG, Shaper G, Walker M. Physical activity and mortality in older men with diagnosed coronary heart disease. Circulation. 2000;102: 1358-63.

75. Hamer M, Stamatakis E. Physical activity and mortality in men and women with diagnosed cardiovascular disease. Eur. J. Cardiovasc. Prev. Rehabil.2009; 16: 156-60.

76. Belardinelli R, Paolini I, Cianci G et al. Exercise Training Intervention After Coronary Angioplasty: the ETICA trial. J. Am. Coll. cardiol. 2001; 37: 1891-1900.

77. Giannuzzi P, Temporelli PL, Corrà $U$ et al. Attenuation of unfavorable remodeling by exercise training in postinfarction patients with left ventricular dysfunction. Circulation. 1997;96: 1790-97. 